\title{
Magnetic ferroelectric materials
}

\author{
H SCHMID \\ Department of Inorganic, Analytical and Applied Chemistry, University of Geneva, 30, \\ Quai Ernest Ansermet, CH-1211 Geneva 4, Switzerland
}

\begin{abstract}
In the form of a succinct overview the structure and symmetry requirements of magnetic ferroelectrics are discussed. Boracites are the best-studied examples and have phases being simultaneously ferroelectric, ferromagnetic and ferroelastic. One of the salient features of such materials is the obligatory occurrence of the linear and bilinear magnetoelectric effects. They represent an invaluable auxiliary information for magnetic symmetry determination by neutron diffraction. Owing to the complexity of property combinations, work with single crystals and polarized light microscopy is obligatory. Key references of the field are given.
\end{abstract}

Keywords. Ferromagnetic ferroelectrics; antiferromagnetic ferroelectrics; ferroelasticity; magnetoelectric effects.

\section{Introduction}

In 1994 we celebrate the centennial of Pierre Curie's idea of a material which can be electrically polarized by means of a magnetic field and magnetized by means of an electric field (Curie 1894). There were many unsuccessful attempts of different scientists at finding such an effect (well chronicled by $\mathrm{O}^{\prime}$ Dell 1970). Finally Landau and Lifshitz remarked that time reversal symmetry has to be taken into account and that the magnetoelectric and piezomagnetic effect should be possible in magnetic structures (Landau and Lifshitz 1960). On that basis Dzyaloshinskii predicted the linear magnetoelectric effect to exist in the antiferromagnetic phase (with Shubnikov point symmetry $\overline{3}^{\prime} \mathrm{m}^{\prime}$ ) of chromium oxide $\mathrm{Cr}_{2} \mathrm{O}_{3}$ (Dzyaloshinskii 1959). Shortly thereafter Astrov confirmed the prediction by measuring the electric field-induced magnetization in $\mathrm{Cr}_{2} \mathrm{O}_{3}$ (Astrov 1960), and the detection of the magnetic fieldinduced polarization followed step (Folen et al 1961; Rado and Folen 1961). These two manifestations of the magnetoelectric effect - measurable in insulators only--can be considered as the two facets of Curie's prediction.

If we take a compensated antiferromagnet like $\mathrm{Cr}_{2} \mathrm{O}_{3}$, permitting the linear magnetoelectric effect, we can alternatively say that owing to the magnetoelectric interaction the material becomes a very weak ferromagnet under the influence of an electric field and a very weak ferroelectric under the influence of a magnetic field. Such a magnetic field-induced ferroelectric is unusual in the sense that its antiferromagnetic domains, which become simultaneously the ferroelectric domains, can be switched by means of an electric field (Martin 1965; Martin and Anderson 1966). We might also speak of an induced electronic ferroelectric because in contrast to common ferroelectrics the nuclear structure remains in first approximation immobile and essentially only the spins of the magnetically active unpaired electrons are reversing during the domain switching process.

Independently of the rigorous symmetry approach by Dzyaloshinskii, Smolensky and Ioffe were trying to synthesize ferroelectric ferromagnets by replacing partially 
diamagnetic ions by paramagnetic ones on the B-site of oxyoctahedral ferroelectric perovskites. By this approach initially the antiferromagnetic ferroelectric perovskite $\mathrm{Pb}\left(\mathrm{Fe}_{1 / 2} \mathrm{Nb}_{1 / 2}\right) \mathrm{O}_{3}$ was synthesized in ceramic form (Smolensky and Ioffe 1958). Later, by studying single crystals, the compound was recognized to have a weak spontaneous magnetic moment in the ferroelectric phase below 9K (Astrov et al 1968). Because of the solid solution approach, implying dilution by diamagnetic ions, the magnetic coupling is, however, substantially reduced.

The simultaneous occurrence of ferroelectricity and (weak) ferromagnetism was first discovered in nickel iodine boracite $\mathrm{Ni}_{3} \mathrm{~B}_{7} \mathrm{O}_{13} \mathrm{I}$ (below $61 \mathrm{~K}$ ) (Ascher et al 1966). This compound is a member of a large crystal structure family with the general formula $\mathrm{M}_{3} \mathrm{~B}_{7} \mathrm{O}_{13} \mathrm{X}$, where $\mathrm{M}$ stands for a bivalent cation of $\mathrm{Mg}, \mathrm{Cr}, \mathrm{Mr}, \mathrm{Fe}, \mathrm{Co}$, $\mathrm{Ni}, \mathrm{Cu}, \mathrm{Zn}$, etc and $\mathrm{X}$ for a monovalent anion like $\mathrm{OH}^{-}, \mathrm{F}^{-}, \mathrm{Cl}^{-}, \mathrm{Br}^{-}, \mathrm{I}^{-}$, or $\mathrm{NO}_{3}^{-}$. Nearly all the boracite compositions with paramagnetic metal ions undergo phase transitions from a cubic high-temperature phase to fully ferroelectric/fully ferroelastic phases (for nomenclature see Aizu 1970), most of which undergo at low temperatures a transition to a (weakly) ferromagnetic phase with orthorhombic, monoclinic or triclinic symmetry (Tolédano et al 1985; Rivera et al 1985).

\section{Conditions for ferromagnetic and antiferromagnetic ferroelectrics}

The conditions for the occurrence of ferroelectricity and magnetic order in the same phase - often accompanied by ferroelasticity (for a definition see Aizu 1970) - comprise (i) the presence of adequate structural building blocks, e.g. with double or multiple potential wells permitting ferroelectric-type ionic movements, (ii) magneticinteraction pathways, usually of the superexchange type, and (iii) the fulfilment of symmetry conditions (Schmid 1973; Ascher 1973; Freeman and Schmid 1975).

Among the 122 Shubnikov-Heesch point groups there are 31 allowing a spontaneous magnetization and 31 allowing a spontaneous polarization. However, the occurrence of both ferro(i)magnetism and ferroelectricity in the same phase is permitted in 13 point groups only (Ascher 1973; Schmid 1973). The presence of both ferroelectricity and antiferromagnetism is allowed in 8 point groups (Schmid 1973); howe ver, this number increases drastically when the possible symmetries of incommensurdte phases are included.

For symmetry reasons all ferro(i)electric ferromagnets allow a fortiori the linear and both bilinear (in the electric field and the magnetic field) type magnetoelectric effects (Ascher 1968; Schmid 1973; Freeman and Schmid 1975). In the boracite family the linear and bilinear (in the magnetic field) magnetoelectric effect has been measured for a variety of compositions (for a bibliography see: Burzo 1994).

For those ferromagnetic ferroelectrics which are simultaneously ferroelastic, a coupling between the spontaneous polarization, spontaneous magnetization and spontaneous deformation is possible. Such a coupling has for the first time been demonstrated for $\mathrm{Ni}_{3} \mathrm{~B}_{7} \mathrm{O}_{13} \mathrm{I}$ (Ascher et al 1966), in which an electric field-induced $180^{\circ}$ reversal of the spontaneous polarization causes a symmetry-conditioned $90^{\circ}$ reorientation of the spontaneous magnetization, and a magnetic field-induced $90^{\circ}$ reorientation of the spontaneous magnetization causes a $180^{\circ}$ reversal of the spontaneous polarization. Such coupling between the spontaneous polarization and the spontaneous magnetization requires ferroelasticity to be allowed and leads to nonlinear effects with hysteresis cycles and memory behaviour, whereas the linear and bilinear (special 
case: quadratic) magnetoelectric effects are in principle non-lossy, thermodynamically reversible effects, if domain switching and possible relaxation phenomena are disregarded.

\section{Examples of ferromagnetic and antiferromagnetic ferroelectrics}

The simultaneous occurrence of ferroelectricity and ferromagnetism has reliably been proved in the boracite family (Toledano et al 1985) and recently in the phosphate $\mathrm{KNiPO}_{4}$ (Lujan et al 1994). The new family of perovskite-orthoferrite solid solutions, $\mathrm{Bi}_{1-x} \mathrm{RE}_{x} \mathrm{FeO}_{3}(\mathrm{RE}=\mathrm{La}, \mathrm{Dy}, \mathrm{Gd})$, with pyroelectric, ferrimagnetic, magnetoelectric phases and Curie temperatures above room temperature, are very promising (Gabbasova et al 1991).

Ferroelectric antiferromagnets like $\mathrm{BiFeO}_{3}, \mathrm{BaMnF}_{4}, \mathrm{REMnO}_{3} \quad(\mathrm{RE}=$ rare earth), etc are more frequent (Schmid 1973; Freeman and Schmid 1975). It is noteworthy that in $\mathrm{BiFeO}_{3}$ and $\mathrm{BaMnF}_{4}$ the linear magnetoelectric effect cancels because of incommensurate structures (Tabares-Munoz et al 1984; Sciau et al 1990). As a consequence the magnetoelectric behaviour wa found to be only that of a non-centrosymmetric paramagnet, as manifested by the bilinear (in the magnetic field) magnetoelectric effect (Tabares-Munoz 1984; Sciau et al 1990). In the large perovskite family many compositions are potential magnetic ferroelectrics (Venevtsev and Gagulin 1994). However, these materials, often known in ceramic form only, are hardly investigated. There is a reason for that: in perovskites and other compounds with a cubic high-temperature prototypic point symmetry $\mathrm{m} \overline{3} \mathrm{~m} 1^{\prime}$ the number of domain states may climb up to 96 for a triclinic ferroelectric ferromagnetic or antiferromagnetic phase. Therefore, when studying such complex ferroelectric magnetics, reliable physical measurements necessitate the availability of single crystals and the preparation of single-domain specimens in conjunction with the indispensable visual control of the domain state by low-temperature and high-temperature polarized light microscopy, both in transmitted and reflected light (Schmid 1993). There is great paucity of such investigations on single crystals of perovskites and other materials up to now.

\section{Applications of the magnetoelectric effect}

One of the important applications of the linear and higher-order magnetoelectric effects, occurring in magnetic ferroelectrics, resides in the fact that they give us precious auxiliary information for determining the magnetic point group, and herewith the magnetic space group, because the Schönflies-Federov space group is usually known from $\mathrm{X}$-ray diffraction work. This means that they provide a precious complementary tool for neutron diffraction, which seldom allows us to determine the magnetic point and space group unequivocally.

Other important scientific applications of the magnetoelectric effect are the accurate determination of magnetic phase-transition temperatures and critical exponents; the study, switching and 'poling' of antiferromagnetic domains by means of simultaneous application of magnetic and electric fields; the study of defects in magnetic phases; magnetic and electric field-induced phase transitions (e.g. spin-flop and metamagnetic transitions); etc (Freeman and Schmid 1975; Schmid et al 1994). 


\section{Conclusions}

In the Proceedings of the 2nd International Conference on Magnetoelectric Interaction Phenomena in Crystals (Schmid et al 1994), the reader will find more information on magnetic ferroelectrics, related materials, their properties, and in particular on theory and experiment of the magnetoelectric effect. This effect represents one of the most salient features of magnetic ferroelectrics.

\section{Acknowledgements}

Warm thanks are due to Prof. S V Suryanarayana, Osmania University, Hyderabad for his invitation to present the paper at the MRSI meeting, Hyderabad and to Shri S L N Acharyulu, DMRL, Hyderabad for stimulation and support. The author is grateful to the Swiss National Science Foundation for supporting research.

\section{References}

Aizu K 1970 Phys. Rev. B2 754

Ascher E 1968 Philos. Mag. 17149

Ascher E, Rieder H, Schmid H and Stössel H 1966 J. Appl. Phys. 371404

Ascher E 1973 Int. J. Magn. 5 287; also in: Freeman and Schmid 1975 p. 69

Astrov D N 1960 Zh. Eksp. Teor. Fiz. 37881 [1960 Sov. Phys.-JE TP 11 708]

Astrov D N, Al'shin B I, Zorin R V and Drobyshev L A 1968 Zh. Eksp. Teor. Fiz. 552122 [1969 Sov. Phys.-JETP 28 1123]

Burzo E 1994 in Numerical data and functional relationships in science and technology (ed.) Landolt-Börnstein (Berlin: Springer) N.S., Group III: Solid State Physics, vol. 27, subvol. h, p. 128-204

Curie P 1894 J. Physique 3e série t.III 393; reprinted 1908 in: Oeuvres de Pierre Curie (Paris: Gauthier-Villars) pp. 136-137

Dzyaloshinskii I E 1959 Zh. Eksp. Teor. Fiz. 37 881 [1960 Sov. Phys.-JETP 37 628]

Folen V J, Rado G T and Stalder E W 1961 Phys. Rev. Lett. 6007

Freeman A J and Schmid H (eds.) 1975 Magnetoelectric interaction phenomena in crystals (London: Gordon \& Breach)

Gabbasova Z V, Kuz' min M D, Zvezdin A K, Dubenko I S, Murashov V A, Rakov D N and Krinetsky I B 1991 Phys. Lett. A158 491

Landau L D and Lifshitz E 1960 Electrodynamics of continuous media (Reading: Addison-Wesley; translation of a Russian edition of 1958)

Lujan M, Rivera J-P, Schmid H, Kizhaev S, Triscone G and Mullar J 1994 Ferroelectrics 16177 (also in Schmid et al 1994, p. 77)

Martin T J 1965 Phys. Lett. 1783

Martin T J and Anderson J C 1966 IEEE Trans. Magn. MAG-2 466

O'Dell T H 1970 The electrodynamics of magneto-electric media (Amsterdam: North-Holland)

Rado G T and Folen V J 1961 Phys. Rev. Lett. 7310

Rivera J-P, Schaefer F-J, Kleemann W and Schmid H 1985 Jpn J. Appl. Phys. 24 Suppl. $24-21060$

Schmid H 1973 Int. J. Magn. 4 337; also in: Freeman and Schmid 1975 p. 121

Schmid H 1993 Polarized light microscopy $(P L M)$ of ferroelectric and ferroelastic domains in Ferroelectric ceramics (eds.) N Setter and E L Colla (Basel: Birkhäuser) pp. 107-126

Schmid H, Janner A, Grimmer H, Rivera J-P and Ye Z-G (eds) 1994 Proc. 2nd Int. Conf. on Magnetoelectric Interaction Phenomena in Crystals (MEIPIC-2) (Gordon and Breach) in: 1994 Ferroelectrics 161-162 $1-745$

Sciau Ph, Clin M, Rivera J-P and Schmid H 1990 Ferroelectrics 105201

Smolensky G and Ioffe V A 1958 Communication No. 71 Colloque International du Magnétisme (Grenoble)

Tabares-Munoz C, Rivera J-P, Bezinges A, Monnier A and Schmid H 1984 Jpn. J. Appl. Phys. 24 Suppl. 24-2 1051

Tolédano P, Schmid H, Clin M and Rivera J P 1985 Phys. Rev. B32 6006

Venevtsev Yu V and Gagulin V V 1994 Ferroelectrics 16223 (also in Schmid et al 1994, p. 371) 